\title{
REDUCTION OF ABLATION PLUME SHADING EFFECT FOR ULTRASHORT PULSED LASER MICROMACHINING OF SILICON
}

\author{
D. Blood ${ }^{l}$, T. Schmitz ${ }^{2}$, and M. Sheplak ${ }^{l}$
}

${ }^{1}$ University of Florida, Gainesville, Florida, USA

${ }^{2}$ University of North Carolina at Charlotte, Charlotte, North Carolina, USA

\section{ABSTRACT}

This paper presents the implementation of a novel technique for increasing the material removal rate (MRR) of silicon by a Nd:YAG ultrashort pulsed laser. During the machining process ablated material can interact with laser pulses to reduce the effective MRR. An analysis of the parameters affecting the ablated materiallaser pulse interaction is explored, along with a technique for increasing the MRR by approximately $177 \%$ and decreasing the surface roughness (Ra) by approximately $65 \%$ over current machining techniques.

\section{INTRODUCTION}

Ultrashort pulsed lasers allow for single step processing of various materials in non-clean room environments [1]. They are advantageous for sensor and actuator fabrication due to their relatively minimal setup requirements, and minimal thermal load imparted into the surrounding workpiece [2]. The traditionally low MRR has thus far inhibited adoption of this technology in industrial applications. Recent increases in maximum laser power output have helped to increase the MRR; however, these advancements have also increased the significance of certain phenomena that negatively affect the machining process. One such phenomena is the ablation plume shading effect.

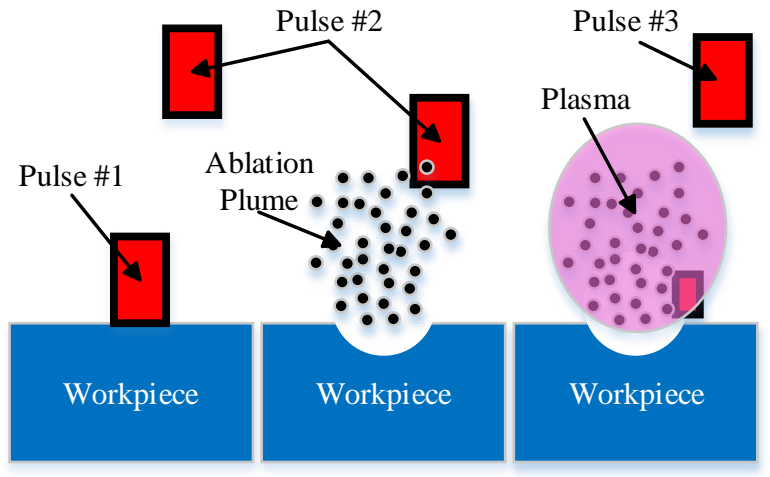

Figure 1: The process of forming plasma over the workpiece surface. First, pulse \#1 impacts the workpiece (left). Then, ablated material is ejected (middle). Lastly, pulse \#2 interacts with the weakly ionized molecules to form a plasma and the pulse intensity is decreased (right).
During the machining process ablated material is ejected out of the cut region [3]. This ejected material can interact with subsequent pulses, as seen in Fig. 1, to produce a plasma above the workpiece [4]. The plasma effectively shades the workpiece from the pulse by absorbing a fraction of the pulse's energy. This shading effect can cause a reduction in the MRR, and an increase in the surface roughness $[5,6]$.

The two primary machining parameters of interest for the ablation plume shading effect are: (1) the pulse area overlap (PAO), and (2) the pulse repetition rate (PRR). A visualization of these parameters is shown in Fig. 2. The PAO affects the distance the plume must dissipate before the subsequent pulse reaches the workpiece, and the PRR affects the time the plume has to dissipate.
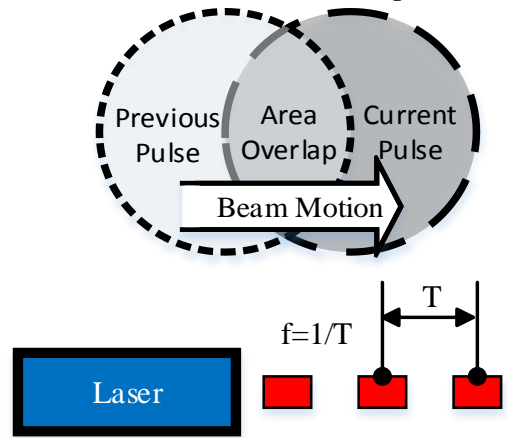

Figure 2: (Above) Visualization of the pulse area overlap, and (Below) the pulse period ' $T$ ' that can be used to calculate the pulse repetition rate ' $f$ '.

\section{EXPERIMENTAL PROCEDURE}

To test the effects of these two parameters, several $100 \mu \mathrm{m}$ squares were machined in <100> P-type silicon by a Coherent Talisker Ultra 355-4 laser with a nominal pulse duration of 10-15 picoseconds, and a wavelength of $355 \mathrm{~nm}$. The results of these tests, shown in Fig. 3, reveal a decreasing trend in the average volume of material removed per pulse for an increasing PRR and an increasing PAO. While decreasing the PRR would reduce the shading effect, it also reduces the overall MRR. Also, decreasing the PAO results in an increase in the surface roughness; consequently, another solution

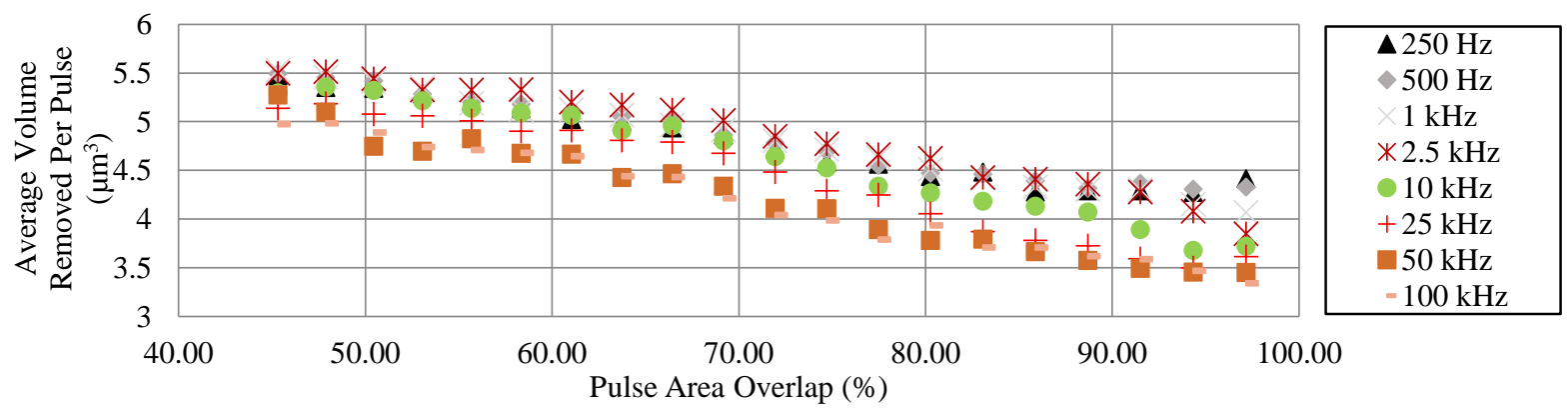

Figure 3: Demonstration that an increase in either the pulse area overlap or the pulse repetition rate results in a decrease in the MRR. 
is necessary to increase the MRR without affecting the quality of the feature.

In an attempt to reduce the effect of the ablation plume shading, various gases were blown over the workpiece surface. It was theorized that a gas flow would help remove the ablated material before the subsequent pulse could interact with the plume, and that a noble gas, in this case argon, would retard the plasma formation. The gases were blown through a $40 \mathrm{~mm}$ wide Lechler air nozzle, shown in Fig. 4, at a flow rate of $40 \mathrm{~L} / \mathrm{min}$, and at a distance of 12.7 $\mathrm{mm}$ from the workpiece surface. Gas flows oriented parallel and perpendicular to the cut direction, as shown in Fig. 5, were tested.

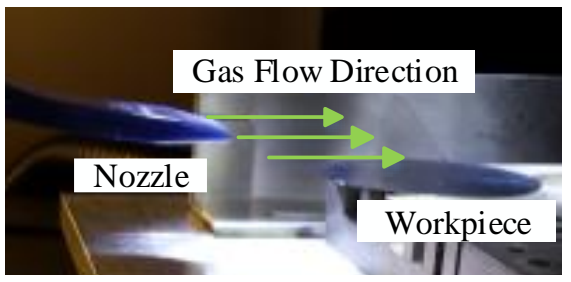

Figure 4: Setup for blowing gasses through a wide airflow nozzle over the silicon workpiece.

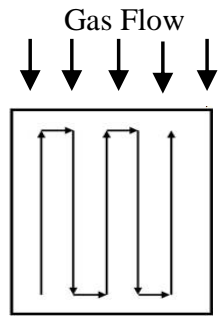

Parallel (\|)

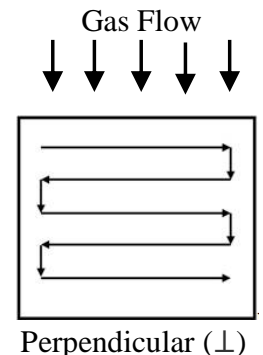

Perpendicular $(\perp)$
Figure 5: Visualization for the direction of gas flow for the two different cases. Lines inside of the squares represent the cut path of the laser.

\section{RESULTS}

Analyzing the results of the tests, shown in Fig. 6, reveals three points. First, the squares machined with a gas flow show greater cut depths for the same number of cut passes; effectively increasing the MRR. Second, the parallel gas flow shows a greater improvement in the cut depth compared to the perpendicular flow. Third, the flow of argon resulted in the largest depth of cut with a $177 \%$ increase over the stagnant machining environment at 19 cut passes.

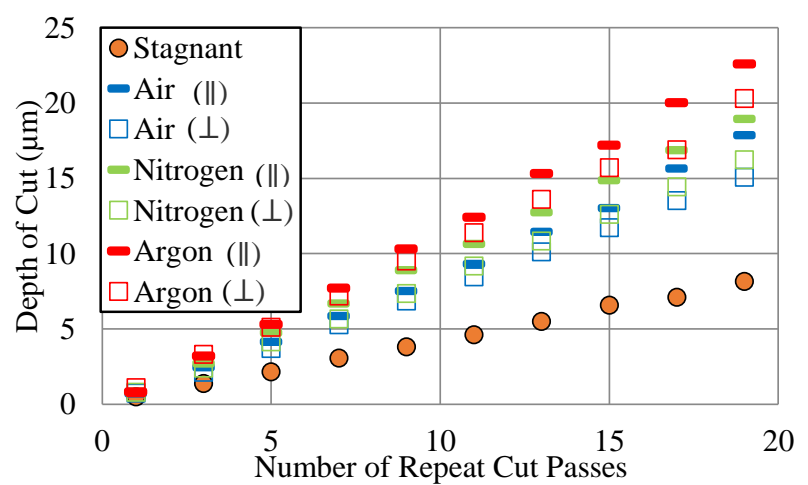

Figure 6: Experimental data validating the theory that gasses blowing over the workpiece can increase the MRR by decreasing the ablation plume shading effect.
In addition to increasing MRR, the surface roughness of the machined workpiece can also be improved with implementation of gas flow. Fig. 7 shows that at a cut depth of $8.16 \mu \mathrm{m}$ the surface roughness $(\mathrm{Ra})$ is $934 \mathrm{~nm}$ for the stagnant condition. With the gas flowing the surface roughness is reduced by approximately $65 \%$ to only $295 \mathrm{~nm}$ at a cut depth of $7.35 \mu \mathrm{m}$.

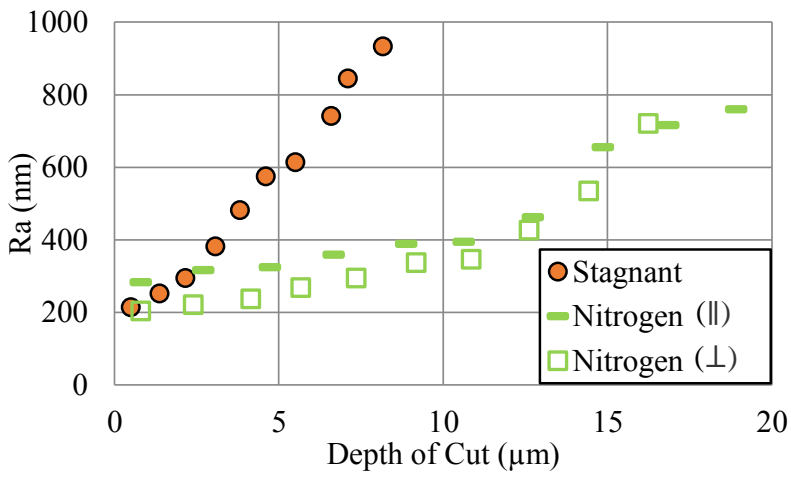

Figure 7: A comparison of the resulting surface roughness for three machining conditions. The presence of nitrogen flowing over the workpiece results in a relatively low surface roughness compared to a stagnant machining condition.

\section{CONCLUSION}

Ultrashort pulsed lasers allow for the machining of silicon in non-clean room environments with minimal setup. Recent advances in laser capabilities increase the potential maximum MRR; however, the ablation plume shading effect is reducing realized gains of these new lasers. While a decrease in the PRR or PAO reduces the effect of this shading, it also results in a lower overall MRR and an increase in the surface roughness. The implementation of a gas flow over the workpiece surface decreases the effect of the ablation plume shading by removing the ablated particles before the subsequent pulse could interact with the plume. Further investigation is necessary in order to optimize the effectiveness of this technique.

\section{ACKNOWLEDGEMENT}

The authors would like to thank the U.S. Department of Energy for their support (DE-FE0012370).

\section{REFERENCES}

[1] N.B. Dahotre, and S.P. Harimkar, Laser Fabrication and Machining of Materials, Springer Science + Business Media, LLC, New York, 2008.

[2] B.N. Chichkov, C. Momma, and S. Nolte, "Femtosecond, Picosecond, and Nanosecond Laser Ablation of Solids," Applied Physics A, 63, pp. 109-115, (1996).

[3] N. Rykalin, A. Uglov, and A. Kokora, Laser Machining and Welding, Pergamon Press Inc., New York, 1978.

[4] N. Dahotre, and A. Samant, Laser Machining of Advanced Materials, Leiden: Yalor \& Francis Group, London, 2011.

[5] D. Bäuerle, Laser Processing and Chemistry, Springer Heidelberg Dordrecht, London, 2011.

[6] S. Amoruso, C. Altucci, and R. Bruzzese, "Study of the Plasma Plume Generated During Near IR Femtosecond Laser Irradiation of Silicon Targets", Applied Physics A, 79, 13771380 (2004).

\section{CONTACT}

*D. Blood, tel: +1-765-366-7835; DANIEL.BLOOD@UFL.EDU

*M. Sheplak, tel: +1-352-392-3983; SHEPLAK@UFL.EDU 